\title{
Applying Quality Index Criterion for Flexible Multi-Detection of Heartbeat using Features of Multimodal Data
}

\author{
Mohammad Javad Mollakazemi, Farhad Asadi, Shadi Ghiasi, S Hossein Sadati \\ Department of Mechanical Engineering at K. N. Toosi University of Technology, Tehran, Iran
}

\begin{abstract}
Introduction: In many conditions contaminated signals and noises can distort electrocardiogram (ECG) signals, so synchronously measured signals could enhance analyzing the heart rate variability. Therefore, the algorithm should be able to identify reliable and optimal segment of these multimodal signals in order to accurately locate the heart beats.

Aims: This paper presents a flexible and multichannel feature extractor and classifier for the purpose of locate heartbeats by using the multi-channel recording from PhysioNet Challenge 2014 database.

Methods: In this study, for feature extraction from multimodal data the common heartbeat detectors are used in other studies are employed, but the exhausted results are promising which indicates the importance and efficiency of the proposed fusion strategy and the decision of when fusion should be done as ECG itself is the best indicator of heartbeat detection. For fusion, after segmentation of waveforms, if ECG assumed to be noisy, an index is assigned to each signal based on periodicity of the extracted features and estimation of noises of detailed coefficients of discrete wavelet transform. The signal whose index is highest is processed in that segment.
\end{abstract}

Results: The presented approach was evaluated by 200 recording of multimodal dataset provided for the PhysioNet/Computing in Cardiology Challenge 2014, and the average accuracy of the $95.39 \%$ was obtained.

\section{Introduction}

In most countries, cardiac disease is one of the top factors of death. Due to its high mortality rate, its accurate and reliable detection is of the utmost importance. Heart disease can be detected via vital signals including Electrocardiogram (ECG), Blood Pressure (BP) and Photoplethysmogram (PPG). Recent advances in computer science particularly in signal processing have made major contributions to automatic analysis of vital signals for cardiac abnormality detection.

Arterial Blood Pressure (ABP) is the outcome of heart's pumping system; all heart abnormalities affect blood pressure and BP signal. Electrocardiogram is a representation of the electrical activity of heart. Different type of noise like power-line interference, baseline drift, electrode contact noise and motion artifacts, degrade the performance of detection process [1].

In recent years, regarding to importance of noise removal from cardiac signals, various techniques have been proposed. The most widely used methods include, linear filtering [2], adaptive filtering, digital filtering, mathematical morphology filtering [3], Neural Networks, independent component analysis, empirical mode decomposition [4], fuzzy logic [5], fixed coefficients filtering [6] and wavelet transform based techniques [7, 8].

This study aims to develop a reliable robust method for detection of exact heartbeat locations in continuous longterm data including electrocardiogram and blood pressure signal based on measuring the signal quality by wavelet and stochastic based techniques.

\section{Material and method}

\subsection{Data}

In this study, 200 recordings of multimodal dataset of PhysioNet/CinC Challenge 2016 were used [9]. Each record contained four to eight signals, the first of which was always an ECG signal. The remaining signals could be any of a variety of simultaneously recorded physiologic signals including blood pressure (BP), arterial line (ART), pulmonary arterial pressure (PAP), and respiration (Resp) which might be useful for robust beat detection.

\subsection{Method}

\subsubsection{Peak detection}

In the proposed method, heartbeats are detected via 
ECG and BP signals, and all the R-waves in ECG and all the existing peaks in the BP signal are detected. In this method, the peak detectors which are used for detection of peaks in ECG and other waveforms are gqrs, Pan \& Tompkins [10] and wabp. It should be noted that gqrs and wabp are the components of WFDB toolbox [11]. Since peaks of blood pressure signal are delayed as compared to the actual heartbeats; this delay (with value of 0.2 seconds) is considered after calculating heartbeats from BP signal so that these heartbeats can be comparable with their corresponding heartbeats omitted from ECG signal.

\subsubsection{Fusion of the multimodal features}

After finding R-waves of ECG and peaks of other waveforms, a window with the length of 5 seconds is slid along both signals. All the detected beats in this window are separately extracted from each signal.

Before fusion, it is better that it be checked whether ECG is noisy or not. Since if ECG is clean and we fuse the features of other signals which themselves are weaker than ECG in reflexing the heart activity, the performance of the algorithm may be reduced significantly. To meet this end, the ECG is segmented to 5 seconds zones, then, in each segment it is checked whether the detections have a correspond pulse in the ABP or not. If all of the R-peaks in a segment of ECG have a detected pulse in ABP signal, and the number of the detections in the segment of ECG and the synchronic segment of ABP is equal, then it is assumed that ECG is clean, and the R-peaks detected in that segment of ECG will be used for final heartbeats. If each heartbeat of ECG does not have a correspond pulse in $A B P$ or the number of detections is not equal in ECG and $\mathrm{ABP}$, then it is assumed that ECG or ABP or both are contaminated with noises. In this case, the features extracted from multimodal signals will be fused.

For fusion, a signal quality index is assigned to each segment based on estimation of noises of detailed coefficients of discrete wavelet transform and the periodicity of the detected features in that segment. The variance of the first derivative of extracted beats is calculated. The variance of derivative of heartbeats is a symbol of the established order between them, and the lower the value of variance the higher is the value of the established order. Therefore, after calculating derivative of detected heartbeats in each signal; heartbeats whose variance is smaller are assigned the higher index for periodicity. In fact, this technique is a noise detection method, as variance of derivative of heartbeats increases in noisy regions of signal, and spontaneously these parts of signal will not be used for recording the final heartbeats.

For ECG the second level of detailed coefficients of db6 is employed for estimation of noise, and for other signals such as ABP, ART, PAP, etc. the second level of detailed coefficients of haar is used. Each segment of each waveform in which the extracted features have higher periodic characteristic will be assigned higher index, and in each segment in which the distribution of noises is lower will be assigned higher index. Afterwards, the assigned indexes from both criteria will be summed, and finally, the features of each segment whose total index is the highest will be used in that zone.

\subsubsection{Post-processing}

In a 5 second comparison of ECG and BP signals, there is probability of having some repeated or undetected beats. These errors are on the border of windows. For instance in a 5 second, the detected beats of ECG are used and in the next 5 second the beats of BP are used. Thus, two incidents are possible:

1- The last heartbeat in a window of ECG is repeated as the first heartbeat in the next 5 seconds window of BP. So, in final beats, a heartbeat is recorded twice (one beat will be redundant).

2- A heartbeat is recorded a few milliseconds after the window of ECG signal is finished; and in the corresponding blood pressure signal, the heartbeat is recorded in the previous window of BP signal (one beat will be missed).

\subsubsection{Filtering redundant beats}

In order to solve the first problem, a filter is designed to omit the additional heartbeats. This filter checks the distance of all beats. Then from every two heartbeats which are closer than minimum of adults R-R interval (0.5 seconds), the one which has lower amplitude in ECG is eliminated.

\subsubsection{Prediction of missed beats}

To resolve the second problem, the position of undetected heartbeat is predicted based on the established order between other heartbeats. Since, most signals are long (10 minutes) and heartbeat rate may vary in different minutes, the location of missing beat is predicted based on the existing order between a few beats before the missing one. There are many other reasons that a heartbeat may be missed, such as existence of noise in Figure 1. Thus, possible location of missing heartbeats in noisy parts of the signal is predicted based on the established order between a few previous heartbeats. So, the prediction part plays a crucial role. 


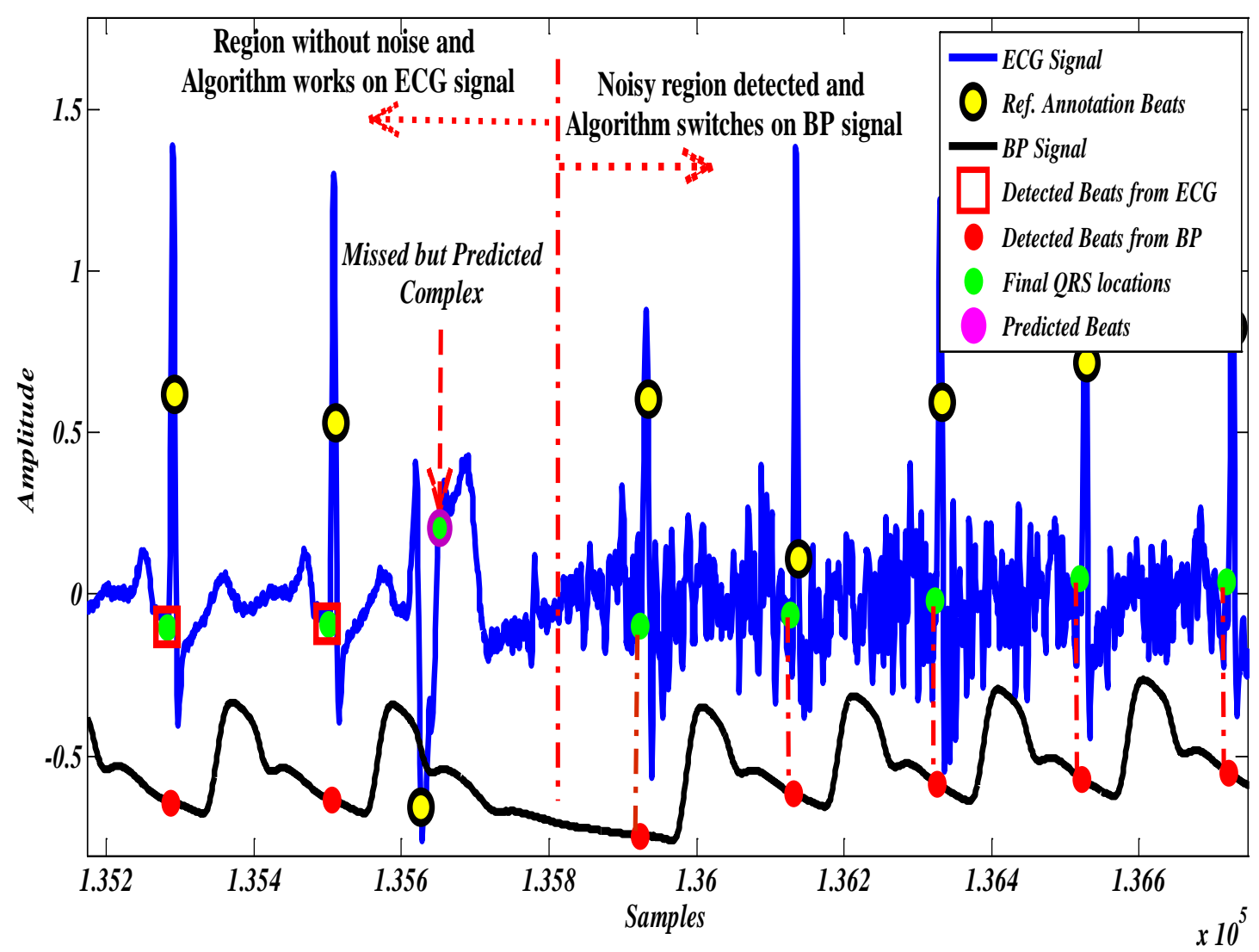

Figure 1. In noisy zones of ECG, BP signal will be used for heartbeat detection instead.

\section{Results}

Three parameters FP, FN, TP are used to express the results. FP (False positive) is the number of wrong heartbeats detected by the method. FN (False negative) is the number of actual heartbeats which are not detected. TP (True positive) is the number of correct heartbeats. To show the method performance, two parameters Se (Sensitivity) and PPV (Positive productivity) are defined as $\mathrm{TP} /(\mathrm{TP}+\mathrm{FN}) * 100$ and $\mathrm{TP} /(\mathrm{TP}+\mathrm{FN}) * 100$ respectively. $\mathrm{Se}$, is the percentage of real beats which are true positive, and PPV is the percentage of detected beats that are true positive. The exhausted results by evaluating on 200 multimodal dataset of PhysioNet/CinC Challenge 2014 are listed in Table 1 and are compared to the other studies as well.

\section{Discussion and conclusion}

This study presents a method to accurately locate heartbeats in multimodal data. According to Table 1, the fusion of the information extracted from multimodal data improves the performance of the method in comparison with individual peak detectors.

The main part of this study is the strategy which is used for fusion of the features extracted from multimodal data. After finding peaks from ECG and ABP, it should be checked whether the ECG is noisy or not. As ECG is the best tool for accurately locate the heartbeats, fusion of the multimodal data is not required when ECG is clean; otherwise it is possible that even the total performance of the algorithm be reduced when ECG is clean and we fuse the features extracted from other waveforms which themselves are noisy.

In noisy zones of ECG, the assigned signal quality index using wavelet and stochastic based methods is measured based on which the algorithm will switch on the other waveforms. If all of them are noisy, the one which is cleaner and more reliable will be used. For instance, in Figure 1, however the R-peaks of the ECG are visible and detectable, the scattering of the wavelet coefficient in the right part of the ECG causes that BP signal seems more reliable by a higher quality index, and it is used for locating the heartbeats instead of that segment of ECG. In the fusion strategy, measuring signal quality by assigning 
an index by using estimation of noises of the detailed coefficients in the discrete wavelet transform, and evaluating the periodicity of the extracted features from multimodal waveforms the noisy parts multimodal signal can be found where the proposed fusion strategy will define the features of which signal should be employed for locating the final heartbeats in that segment.

Table 1. The exhausted results of the proposed method.

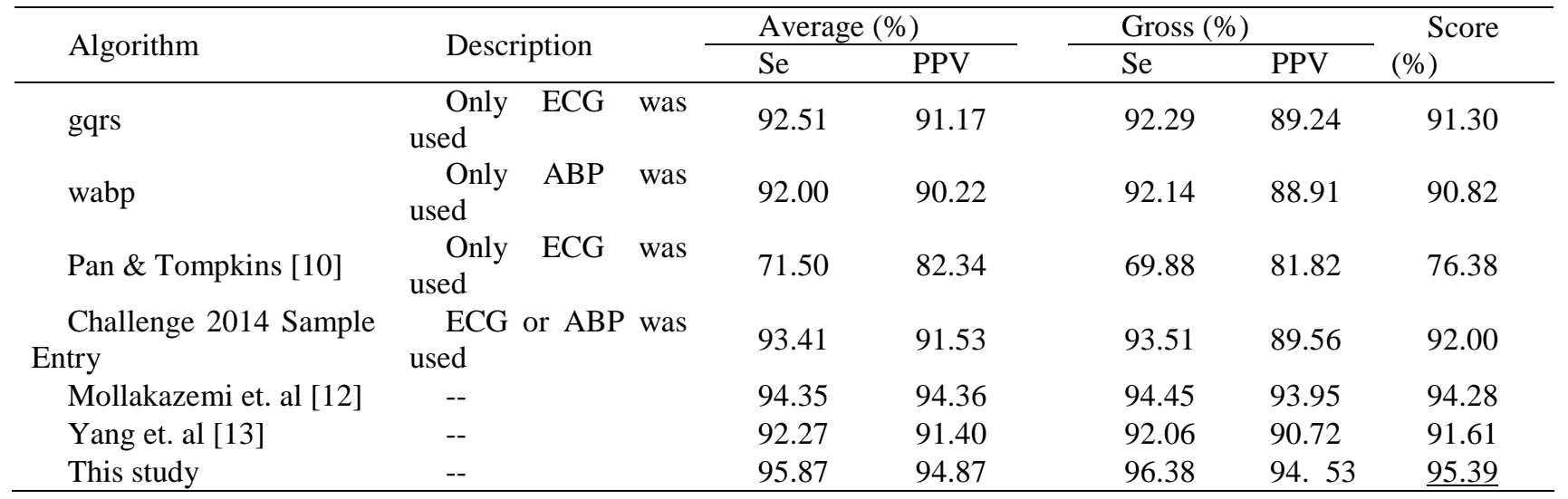

\section{References}

[1] Ghongade R, Ghatol A. A novel QRS detection method.International Journal of Applied Computing 2009;2:6-13.

[2] Von R, Pierluissi J, Nazeran H. Wavelet transform based ECG baseline drift removal for body surface potential mapping. Engineering in Medicine and Biology Society 2005;4:3891-4.

[3] Zhang W, Li M, Zhao J. Research on electrocardiogram signal noise reduction based on wavelet multi-resolution analysis. Wavelet analysis and pattern recognition 2010; 351-4.

[4] Samadi S, Shamsollahi MB. ECG noise reduction using empirical mode decomposition based on combination of instantaneous half period and soft-thresholding. Biomedical engineering (MECBME) 2014;244-8.

[5] Joshi S, Vatti R, Tornekar R. A survey on ECG signal denoising techniques. Communication Systems and Network Technologies 2013;60-4.

[6] Raita-aho T, Saramaki T, Vainio O. A Digital filter chip for ECG signal processing. Instrumentation and Measurement 1994;43:644-9.

[7] Ghaffari A, Mollakazemi MJ, Atyabi SA, Niknazar M. Robust fetal QRS detection from noninvasive abdominal electrocardiogram based on channel selection and simultaneous multichannel processing. Australasian Physical \& Engineering Sciences in Medicine 2015; 38(4):581-92.

[8] Asadi F, Mollakazemi MJ, Uzelac IL, Moosavian SA. A novel method for arterial blood pressure pulse detection based on a new coupling strategy and discrete wavelet transform. Computing in Cardiology 2015; 42:1081-84.

[9] Silva I, Moody B, Behar J, Johnson A, Oster J, Clifford GD, Moody GB. Robust detection of heart beats in multimodal data. Physiological measurement 2015; 36(8):1629.

[10] Pan J, Tompkins WJ. A real-time QRS detection algorithm. IEEE Trans. Biomed. Eng 1985; 32:230-36.

[11] Goldberger AL, Amaral LA, Glass L, Hausdorff JM, Ivanov PC, Mark RG, Mietus JE, Moody GB, Peng CK and Stanley H E. Physiobank, physiotoolkit, and PhysioNet components of a new research resource for complex physiologic signals. Circulation 2000; 101:e21520

[12] Mollakazemi MJ, Atyabi SA, Ghaffari A. Heart beat detection using a multimodal data coupling method. Physiological measurement 2015; 36(8):1729.

[13] Yang B, Teo SK, Hoeben B, Monterola C, Su Y. Robust identification of heartbeats with blood pressure signals and noise detection. Computing in Cardiology 2014; 41: 565568.

Address for correspondence:

Mohammad Javad Mollakazemi

molakazemi.javad@yahoo.com

K.N. Toosi University of Technology, No. 15, Pardis Street, MolaSadra Avenue, Vanak Sq.,Tehran, Iran. 